Canad. Math. Bull. Vol. 23 (4), 1980

\title{
A NOTE ON THE UNIVERSE OF A CATEGORY OF FRACTIONS
}

\author{
BY \\ SRIBATSA NANDA
}

Let $\mathscr{C}$ be a small $\mathcal{U}$-category where $\mathcal{U}$ is a fixed Grothendieck universe, i.e., the objects of $\mathscr{C}$ form a set which is a subset of $\mathscr{U}$ and, for every pair of objects $X, Y$ of $\mathscr{C}$, the set $\mathscr{C}(X, Y))$ is an element of $\mathscr{U}$. If $S$ is a set of morphisms of $\mathscr{C}$, then, in general, the category of fractions $\mathscr{C}\left[S^{-1}\right]$ would belong to a higher universe ([4], p. 256). This presents some difficulties; for example, if $\mathscr{C}$ is the category of based $C W$-complexes and homotopy classes of maps, then for a fixed object $X$ of $\mathscr{C}$,

$$
\mathscr{C}\left[S^{-1}\right](-, X): \mathscr{C} \rightarrow \text { Sets }
$$

defines a homotopy functor and one would like to know if this functor is representable. In order to be able to use Brown's representability theorem ([5], p. 157), one would like to have that the category of fractions should also belong to the same initial universe. Deleanu, in his papers $[1,2]$ has assumed a certain condition (e.g., condition $\left(^{*}\right)$ in [1]), which precisely has the role of guaranteeing that the category of fractions remains within the given initial universe. In this note, we show that if the set of morphisms $S$ is 'good' enough, then $\mathscr{C}\left[S^{-1}\right]$ is a small $\mathcal{U}$-category or, equivalently that condition $\left(^{*}\right)$ of Deleanu is satisfied. Thus we show that under certain conditions, no change of universe is necessary when one forms the category of fractions. This increases the applicability of Deleanu's theorem (see the main theorem of [2]) on the existence of the Adams completion of an object in a cocomplete category. We prove the following

Proposition. Let $\mathscr{C}$ be a small $\mathscr{U}$-category and $S$ a set of morphisms of $\mathscr{C}$ that admits a calculus of left fractions. Then $\mathscr{C}\left[\mathrm{S}^{-1}\right]$ is a small U-category.

Proof. Let $Y$ be a fixed object of $\mathscr{C}$. Denote by $S_{Y}$ the set of morphisms $\left\{s: Y \rightarrow Y^{\prime}, s \in S, Y^{\prime}\right.$ an object of $\left.\mathscr{C}\right\}$. For any object $Y^{\prime}$ of $\mathscr{C}$, let $S_{Y, Y^{\prime}}=$ $\left\{s: Y \rightarrow Y^{\prime}, s \in S\right\}$. It is clear that

and

$$
S_{Y}=\bigcup_{Y^{\prime}} S_{Y, Y^{\prime}}
$$

$$
S_{Y, Y^{\prime}}=S \cap \mathscr{C}\left(Y, Y^{\prime}\right)
$$

Received by the editors September 16, 1978 and, in revised form, March 20, 1979. 
Since $\mathscr{C}$ is a small $\mathscr{U}$-category, $\mathscr{C}\left(Y, Y^{\prime}\right)$ belong to $\mathscr{C}$ and so does $S_{Y, Y^{\prime}}$ being a subset of $\mathscr{C}\left(Y, Y^{\prime}\right)$. Therefore, the set $S_{Y}$, being a union of sets all belonging to $\mathcal{U}$ and indexed by the objects $Y^{\prime \prime}$ of $\mathscr{C}$ (which is a subset of $\mathcal{u}$ ) is itself in $\mathcal{u}$.

Now recall that since $S$ admits a calculus of left fractions, every morphism in $\mathscr{C}\left[S^{-1}\right](X, Y)$ can be represented by a pair $(f, s)$ with $s \in S$ and $f$ a morphism of $\mathscr{C}:$

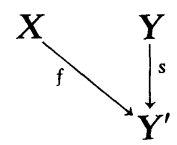

Two such pairs $(f, s)$ and $(g, t)$ are equivalent if there exist morphisms $a$ and $b$ in $\mathscr{C}$ such that

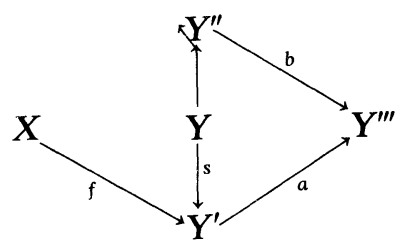

$a s=b t \in S$ and $a f=b g$. We now fix our objects $X$ and $Y$. It is clear that every element of $\mathscr{C}\left[S^{-1}\right](X, Y)$ is represented by a pair $(f, s)$ with $s \in S_{Y}$; moreover, for a given $s: Y \rightarrow Y^{\prime}$ in $S_{Y}$, the set of $(f, s)$ with fixed second coordinate $s$ is in bijective correspondence with the set $\mathscr{C}\left(X, Y^{\prime}\right)$. Thus the set of all such pairs $(f, s)$ is in bijective correspondence with the set $\bigcup \mathscr{C}\left(X, Y^{\prime}\right)$, the union being taken over $S_{Y}$. Since each of these sets is in $U$ and the index set is in $\mathcal{U}$, it follows from the axioms of a Grothendieck universe that the union also belongs to $\mathscr{U}$. Since the objects of $\mathscr{C}\left[S^{-1}\right]$ are same as those the category $\mathscr{C}$, it follows that $\mathscr{C}\left[S^{-1}\right]$ is a small $\mathscr{U}$-category and our proof is complete.

Remark 1. Now recall the following condition $\left(^{*}\right)$ of Deleanu [1, 2]: For a given object $Y$ of $\mathscr{C}$, there exists a subset $T_{Y}$ of the set $S_{Y}=\left\{s: Y \rightarrow Y^{\prime}, s \in S\right.$, $Y^{\prime}$ any object of $\mathscr{C}$ \} such that (i) $T_{Y}$ is an element of $\mathscr{U}$ and (ii) for each $s: Y \rightarrow Y^{\prime}$ in $S$, there is a morphism $s^{\prime} \in T_{Y}$ and a morphism $u$ of $\mathscr{C}$ such that $u s=s^{\prime} ;$ thus, there is a commutative diagram.

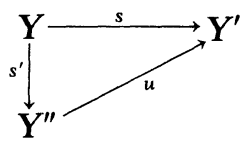

Under the assumptions of the Proposition, $S_{Y}$ is an element of the universe $\boldsymbol{u}$; we may, therefore, take $T_{Y}=S_{Y}$. In that case, given $s: Y \rightarrow Y^{\prime}$ in $S$, we take $s^{\prime}=s$ and $u=i d_{Y^{\prime}}$, thus giving rise to a commutative diagram

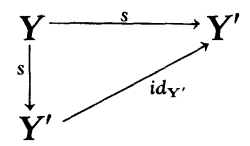


Condition $\left({ }^{*}\right)$ is thus automatically satisfied. In view of this, Deleanu's theorem on the existence of the Adams completion [2] will read as follows:

THEOREM 1. Let $\mathscr{C}$ be a cocomplete small $\mathscr{U}$-category and $S$ a set of morphisms of $\mathscr{C}$ which is saturated and which, moreover, admits a calculus of left fractions. Assume that the following condition on compatibility with coproducts is satisfied: If each $s_{i}: X_{i} \rightarrow Y_{i}, i \in I$, is an element of $S$ where the index set $I$ is an element of $\mathcal{U}$, then $\amalg s_{i}: \amalg X_{i} \rightarrow \amalg Y_{i}$ is an element of $S$. Then every object of $\mathscr{C}$ has an Adams completion with respect to the set of morphisms $S$.

Remark 2. All the arguments above can, of course, be dualised and in view of the application [3], we state the dual result specifically.

THEOREM 2. Let $\mathscr{C}$ be a complete small $U$-category and $S$ a set of morphisms of $\mathscr{C}$ which is saturated and which, moreover, admits a calculus of right fractions. Assume that the following condition on compatibility with products is satisfied: If each $s_{i}: X_{i} \rightarrow Y_{i}, i \in I$, is an element of $S$ where the index set $I$ is an element of $\mathcal{U}$, then $\Pi s_{i}: \Pi X_{i} \rightarrow \Pi Y_{i}$ is an element of $S$. Then every object of $\mathscr{C}$ has an Adams cocompletion with respect to the set of morphisms $S$.

It is a pleasure to thank Professor J. F. Adams for kindly going through the proposition and to the referee for his comments which resulted in an improved presentation of the paper.

\section{REFERENCES}

1. A. Deleanu, Existence of the Adams completion for CW-complexes, J. Pure and App. Alg. 4 (1974), 299-308.

2. A Deleanu, Existence of the Adams completion for objects of cocomplete categories, J. Pure and App. Alg. 6 (1975), 31-39.

3. S. Nanda, Adams cocompletion and the minimal model (preprint).

4. H. Schubert, Categories, Springer, Berlin (1972).

5. R. M. Switzer, Algebraic Topology-Homotopy and Homology, Springer, Berlin (1975).

MATHEMATICS DepartMENT

Regional Engineering College

ROURKELA-8 (ORISSA), INDIA 769008 